\title{
REDUCTION IN INFLAMMATORY MARKERS EXPRESSION IN SERUM IS RELATED TO IMPROVEMENT IN RENAL FUNCTIONS IN INTRAUTERINE UNDERNOURISHED RATS
}

\author{
M. Landgraf ${ }^{1}$, A.E. Hirata $^{2}$, R.G. Landgraf ${ }^{3}$, M.C. Costa $^{1}$, D.T.K. de Marco $^{2}$, P. Semedo ${ }^{4}$, D.A.G. Vieira ${ }^{2}$, \\ F.Z. Gil ${ }^{2}$, N.O.S. Camara ${ }^{1}$ \\ ${ }^{1}$ Immunology, Institute of Biomedical Sciences - University of São Paulo, ${ }^{2}$ Dept of Physiology, Federal \\ University of São Paulo, ${ }^{3}$ Dept of Biological Sciences, Federal University of São Paulo - Campus Diadema, \\ ${ }^{4}$ Dept of Nephrology, Federal University of São Paulo, São Paulo, Brazil
}

Background and aims: Maternal undernutrition can induce a range of fetal adaptations, which can lead to permanent alterations in adulthood. Interleukin (IL)-18 play an integral role in tubular injury and the development of renal dysfunction during a variety of inflammatory processes. In this work, we have investigated the impact of intrauterine undernutrition on the inflammatory markers, and the correlation of these markers with the renal dysfunction; the effect of L-arginine (L-arg)administration on those parameters also was investigated.

Methods: Female Wistar rats were randomly divided into 2 groups: nourished(NR-ad libitum diet) and undernourished (UR-50\% food restriction). After birth, each litter was left with the mother for 28 days. Some of the offspring received a $2 \%$ L-Arg solution in drinking water (groups NR+L-Arg and UR+L-Arg). Proteinuria and glomerular filtration rate (GFR) were determined. IL-18 and cytokine induced neutrophil chemoattractant-2 (CINC-2) were evaluated by Bioplex, in serum.

Results: In UR, the proteinuria (63\%), IL-18 (121\%) and CINC-2 (61\%) were increased, when compared to NR, and the L-arg treatment abolished these effects; the GFR was decreased in UR (43\%), and the L-arg treatment prevented this reduction.

Conclusions: In UR, the inflammatory markers, such as IL-18, are present in an early stage after birth, probably contributing for the development of renal injury in this model. The lower expression of these markers seems to be directly related to improvement of renal function. Our results suggest that these inflammatory markers can be attenuated by L-arginine.

Supported by FAPESP, CNPq, and INCT Complex Fluids 